\title{
Research on Image Processing System for Retinal Prosthesis
}

\author{
Wei Mao ${ }^{1, \mathrm{a}}$, Dashun Que ${ }^{2, \mathrm{~b}}$, Huawei Chen ${ }^{1}$, Mian Yao ${ }^{1}$ \\ ${ }^{I}$ School of Information Engineering, Wuhan University of Technology, \\ Wuhan, Hubei 430070, China \\ ${ }^{2}$ Key Laboratory of Fiber Optic Sensing Technology and Information \\ Processing, Wuhan University of Technology, Ministry of Education, \\ Wuhan, Hubei 430070, China \\ awei_mao_whut@163.com,bdsque@sina.com
}

\begin{abstract}
.
With the development of biomedical engineering, computer science and the bionics, medical treatment develops fast with them too, because these technologies are widely used in medical treatment. Retinal prosthesis is an example based on these technologies. In this paper Spartan 6 series XC6SLX150T development board is used as the hardware platform, combining ISE12.2 with MATLAB as the software development environment. The simulation and design of image processing system for visual prosthesis is done, $32 \times 32$ pixels high resolution retinal prosthesis image processing is achieved. Simulation results prove that this system has a high resolution and good real-time characteristic, which can be an example of image processing system for retinal prosthesis based on FPGA.
\end{abstract}

Keywords: Retinal prosthesis; Image processing; MATLAB; FPGA

\section{Introduction}


Retinitis pigmentosa(RP) and age-related macular degeneration(AMD) are two main diseases that cause the deterioration in vision and even lead to blindness. Retinal prosthesis is mainly applied to visual impairment and blindness caused by them[1].Retinal prosthesis is a kind of artificial organ that can replace retina and make blind people recovering their sight by the implanted electronic micro stimulator affecting the visual nervous system. The electrical stimulation on neurons of the visual system can visualize the signal of the retinal prosthesis. Visual impairment is a major disability faced by millions around the world [2]. According to a statistical report released by the U.N., there are about 37 millions of people suffering from blindness, and about 161 millions more suffering from the deterioration in vision by 2004[3].

In this paper Xilinx Spartan 6 series XC6SLX150T-3FGG676 development board and two daughter boards (FMC-DVI and FMC-IMAGEOV) are used as the hardware platform. The software development environment is ISE12.2, Xilinx Platform Studio and MATLAB2010B. At last we implement the system, and get a better visual effect.

The principle and structure of the image processing system for retinal prosthesis

Although the special structure of all kinds of retinal prosthesis is different, but the modules of the system is same. A typical retinal prosthesis system structure diagram is shown in figure 1.

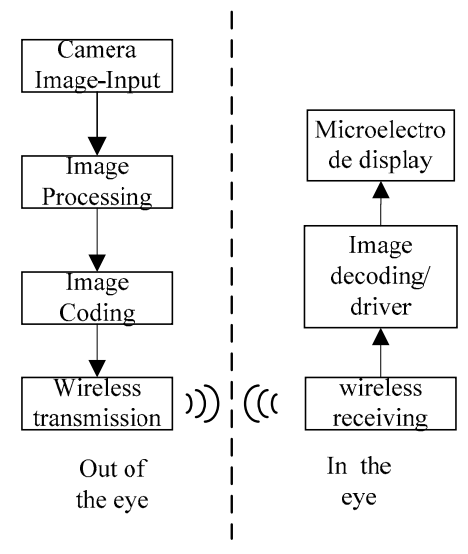


Fig.1 The system structure diagram of retinal prosthesis

The structure of retinal prosthesis system is divided into two parts: the inside eye part and the outside the eye part .The outside eye part responsible for image acquisition, image preprocessing, coding and wireless launcher. The inside eye part includes wireless receiver, implanted nerve stimulator and the microelectrode array. The outside eye part we collect the image information by an external micro video camera. After the image processing module, coded the image applicable for transmitting and stimulating. Then send them into launching module; Intraocular part of retinal prosthesis ,the wireless receiver receives signals from the outside eye part and send them to the micro electric current stimulator, the micro electric current stimulator will load the multi-channel electrical impulses to microelectrode array and stimulate the optic nerve system, so as to realize the function of visual illusion.

The image processing part is the research focus. Perspective transformation is the first step of image processing, and then to determine the image whether need to be enhanced on the basis of visual effect. Subsequent is edge extraction, Canny and Sobel operator are used in retinal prosthesis image processing[4]. Canny operator has a better reaction details, but more time-consuming, so we choose the Sobel operator. Sobel operator uses the gray value of up, down, left and right side of a pixel to detect the edges. The formula is:

$S_{x}=\{f(x+1, y-1)+2 f(x+1, y)+f(x+1, y+1)\}-\{f(x-1, y-1)+2 f(x-1, y)+f(x-1, y+1)\}$

$\mathrm{S}_{\mathrm{y}}=\{(\mathrm{x}+1, \mathrm{y}+1)+2 f(\mathrm{x}, \mathrm{y}+1)+f(\mathrm{x}-1, \mathrm{y}+1)\}-\{(\mathrm{x}+1, \mathrm{y}-1)+2 f(\mathrm{x}, \mathrm{y}-1)+f(\mathrm{x}-1, \mathrm{y}-1)\}$

The combination of $\mathrm{S}_{x}$ and $\mathrm{S}_{\mathrm{y}}$ is the image gradient:

$$
S=\sqrt{s_{x}^{2}+s_{y}^{2}}
$$

After extracting the edge, the edge is often thin, and there are fractures in some places, so the image need for expansion. Because the number of electrodes implanted intraocular is limited, the image should be carried out into low pixels. 
After low pixelated the image is only $32 \times 32$ pixels in size, it is not conducive to watch. Although can be amplified, still it can't simulate the effect displayed on the microelectrode, so finally phosphene simulation is needed.

Phosphene simulation algorithm: (1) build a black background of the same size of the original image; (2)divided it into $32 \times 32$ blocks, build a $3 \times 3$ block matrix in the most left and highest part of the image, pick the most middle block of $3 \times 3$ block matrix, we call it block $\mathrm{Ni}$, the grey value of $\mathrm{Ni}$ is equal to the grey value of the pixel middle of the low pixelated $3 \times 3$ pixel bitmap, the remaining the gray value of eight other blocks is 0 ; (3) use $3 \times 3$ block matrix scan the whole image in turn.

The MATLAB Simulation and FPGA Implementation of Image Processing for Retinal Prosthesis

\section{The MATLAB Simulation Results}

In order to verify the processing effect, MATLAB simulation is conducted. Figure 2 reveals the procedures of image processing.

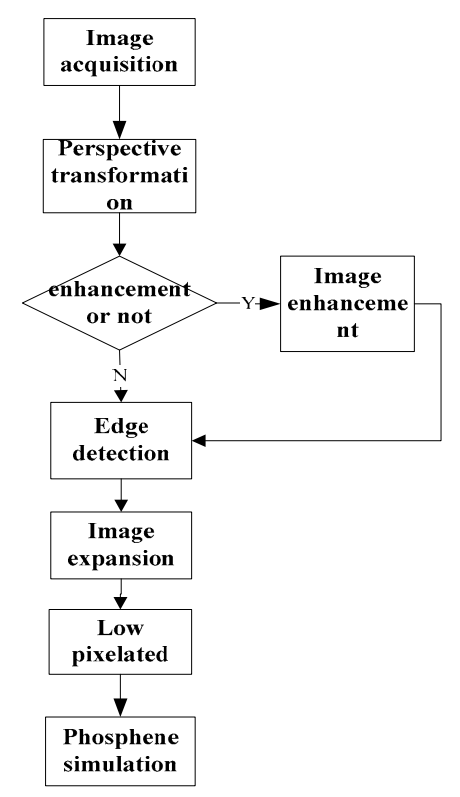


Fig2. The diagram of retinal prosthesis image processing part

The first step involves the rectification of visual angle to get the right middle part of visual angle $20^{\circ} \times 20^{\circ}$ [5]. The second step performs the enhancement of image. The third step involves edge extraction. The image should be low pixelizated to make each pixels corresponding to a micro electrode at the same location. In this experiment, the final dot array effect simulating image is achieved by low pixelization and phosphene simulation.

In this research we elected $32 \times 32$ as parameters, so eventually the image should be processed into $32 \times 32$ pixels. Through low pixel algorithm and phosphene simulation algorithm, finally we get the lattice simulation diagram. As shown in figure 3 :

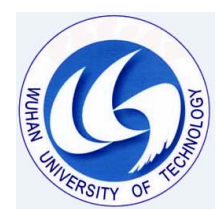

(a)

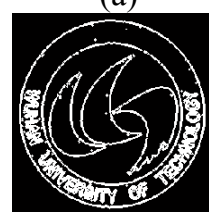

(d)

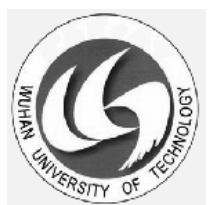

(b)

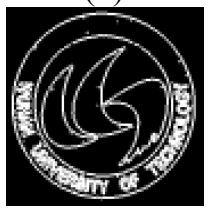

(e)

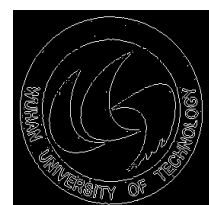

(c)

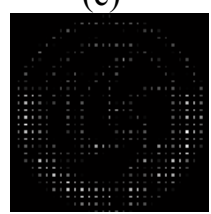

(f)

Figure3 The effect of various steps in the process of MATLAB processing. (a) Adjusted original image (b) image enhancement (c)Image edge detection (d) Image expansion (e) low pixelated to 32 x 32 (f) lattice simulation

\section{Retinal Prosthesis Image Processing System on FPGA}

The structure diagram of retinal prosthesis image processing system on FPGA is shown in figure 4, the camera is used to collect images, through each part of the video signal processing, finally the processed images are displayed on the computer monitor. 


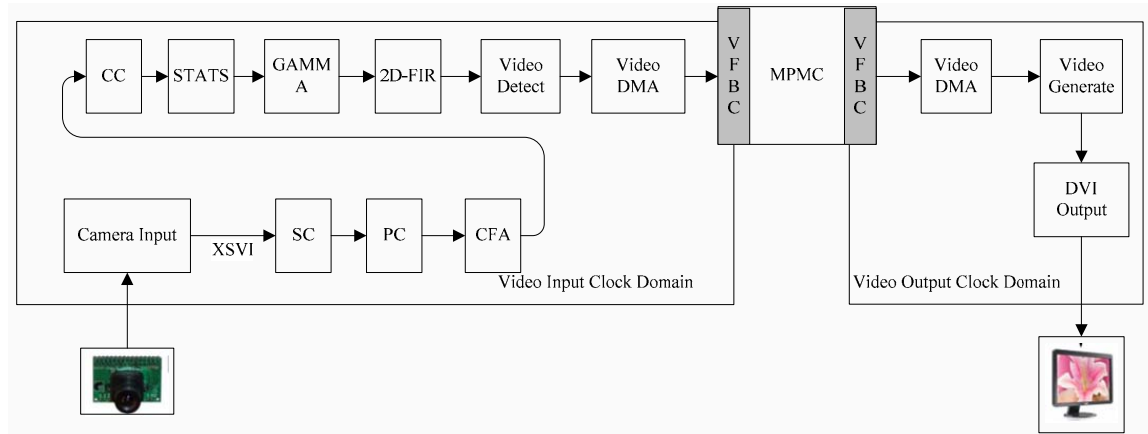

Fig.4 The structure diagram of retinal prosthesis image processing system on FPGA

Image processing part focuses on the design of $2 \mathrm{~d}$ - FIR filter, we using $5 \times 5$ convolution template to design IP core in the System Generator[6], and then simulated it in the in the EDK Xilinx Platform Studio. At last we downloaded it to the FPGA development board, the final processing effect is shown on the monitor. The image processing steps involved in FPGA is still the same as in the MATLAB [7]. At the same time, we compared the visual effect under different resolutions. As shown in figure5:

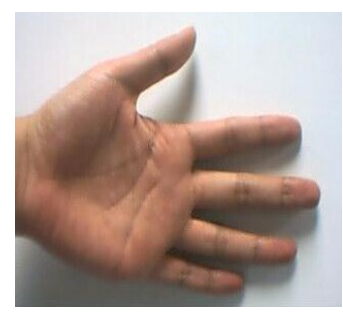

(a)

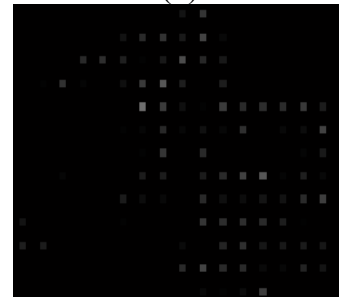

(c)

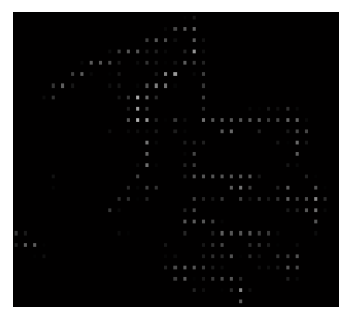

(b)

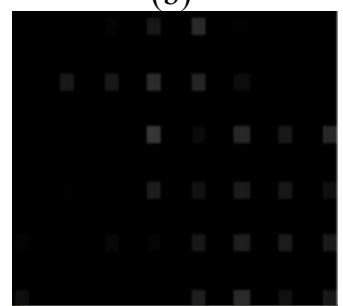

(d)

Fig.5 Visual Effect under Different Resolutions (a) the original image; (b) $32 \mathrm{x}$ 32 dot matrix simulation; (c) $16 \times 16$ dot matrix simulation; (d) $8 \times 8$ dot matrix simulation. 
According to the picture we can see $32 \times 32$ dot matrix has a higher resolution and more conducive to be recognized. It can effectively help blind people to avoid some dangers, and make more convenience for their daily life.

\section{Conclusions}

In this paper, an image processing system for retinal prosthesis based on FPGA is designed, relevant simulations on MATLAB are done and retinal prosthesis image processing algorithms are implemented, which mainly includes the transformation of visual angle, edge extraction, image dilation, low pixelation and the simulation of lattice effects. Experiments show that the image processing system has higher resolution and real-time effect.

\section{References}

[1] Hao Wu, Jing Wang, Heng Li, Xinyu Chai,Prosthetic Vision Simulating System and Its Application Based on Retinal Prosthesis[C], Information Science, Electronics and Electrical Engineering (ISEEE) 2014 International Conference on, Vol.1(2014),p.425 - 429.

[2] Weiland, J.D.Humayun, M.S. Tanguay, A.R.,Out of Darkness: Helping the Blind See with Artificial Vision , Solid-State Circuits Magazine, IEEE, Vol.4-2( 2012),p. $43-45$.

[3] Resnikoff S, Pascolini D, Etya'ale D, Kocur I, Pararajasegaram R, Pokharel GP, Mariotti SP. Global data on visual impairment in the year 2002 [J]. Bull World Health Organ, Vol.11(2004),p. 844-851.

[4] Snaith M, Lee D, Probert P. A low-cost system using sparse vision for navigation in the urban environment [J]. Image and Vision Computing, Vol.4(1998),p.225-233.

[5] Weiland JD, Humayun MS. Visual prosthesis [J]. Proceedings of the IEEE, Vol.7: (2008),p. 1076-1084.

[6]Xilinx,System Generaot for DSP version 12.1 user's guide(2010).

[7] Donald G. Bailey,Design for Embedded Image Processing on 
FPGAs, Wiley-IEEE Press(2011). 